\title{
Ecologías de aprendizaje en educación secundaria: TIC y aprendizaje informal
}

\begin{abstract}
Rebeca Mariel Martinenco
Becaria doctoral del Consejo Nacional de Investigaciones Científicas y Técnicas (CONICET) en el Instituto Académico Pedagógico de Ciencias Humanas de la Universidad Nacional de Villa María rebeca_martinenco@hotmail.com | https://orcid.org/0000-0002-2781-3381
\end{abstract}

Rocío Belén Martín

Investigadora asistente del CONICET en el Centro de Investigaciones y Transferencia de Villa Maríal Profesora adjunta en el Departamento de Enseñanza de la Ciencia y la Tecnología de la Facultad de

Ciencias Exactas, Físicas y Naturales de la Universidad Nacional de Córdoba rbmartin@unc.edu.ar | https://orcid.org/0000-0003-3172-0070

Leticia García Romano

Investigadora asistente del CONICET/Profesora titular en el Departamento de Enseñanza de la Ciencia y la Tecnología de la Facultad de Ciencias Exactas, Físicas y Naturales de la Universidad Nacional de Córdoba leticia.garcia@unc.edu.ar | https://orcid.org/0000-0003-3552-0287

\section{Extracto}

Actualmente se sabe que los estudiantes aprenden en diversos contextos y haciendo un amplio uso de las tecnologías de la información y la comunicación (TIC) (Maina y García, 2016). En el presente trabajo nos proponemos identificar qué aplicaciones, plataformas web, redes sociales y tecnologías digitales utiliza un grupo de estudiantes de educación secundaria mediante un cuestionario autoadministrado. En primer lugar, haremos referencia al concepto de "ecologías de aprendizaje» y a su vinculación con el aprendizaje informal y las TIC. El apartado de «Material y métodos» recoge la caracterización de los participantes y una descripción detallada del instrumento utilizado. Desde un enfoque cualitativo, los resultados se organizan en cuatro categorías. A modo de discusión, planteamos la incidencia de las tecnologías en múltiples aspectos de la vida de los participantes.

Palabras clave: ecologías de aprendizaje; tecnologías de la información y la comunicación (TIC); aprendizaje informal; educación secundaria; cuestionario; contexto de aprendizaje.

Fecha de entrada: 11-06-2020 / Fecha de revisión: 28-06-2020 / Fecha de aceptación: 01-07-2020

Cómo citar: Martinenco, R. M., Martín, R. B. y García Romano, L. (2021). Ecologías de aprendizaje en educación secundaria: TIC y aprendizaje informal. Tecnología, Ciencia y Educación, 18, 77-97. 


\title{
Learning ecologies in secondary education: ICT and informal learning
}

\author{
Rebeca Mariel Martinenco \\ Rocío Belén Martín \\ Leticia García Romano
}

\begin{abstract}
It is known that currently the students learn in different contexts and with a big use of information and communication technologies (ICT) (Maina and García, 2016). In this work we aim to identify wich applications, web platforms, social networks and digital technologies a group of secondary education students uses, through a self-administrated questionnaire. First, we will refer to «learning ecologies» concept and its link with informal learning and ICT. The «Material and methods" section includes the participants characterization and a detailed description of the instrument used. Results are organized in four categories. In the discussion, we propose the impact of technologies on several aspects in the participants' lives.
\end{abstract}

Keywords: ecologies of learning; information and communication technologies (ICT); informal learning; secondary education; questionnaire; learning environment. 


\section{Sumario}

1. Introducción y estado de la cuestión

2. Ecologías de aprendizaje

2.1. TIC y aprendizaje informal

3. Material y métodos

3.1. Participantes

3.2. Instrumento

3.3. Procedimientos

4. Análisis y resultados

4.1. Usos generales del teléfono móvil

4.2. Empleo de aplicaciones y redes

4.3. Uso de tecnologías en el hogar

4.4. Uso de tecnologías en las clases

5. Discusión y conclusiones

Referencias bibliográficas 


\section{Introducción y estado de la cuestión}

Son diversas las historias que las personas tienen en relación a sus ecologías de aprendizaje. Matt Groening, el creador de Los Simpson, creció rodeado por las ilustraciones que realizaba su padre, un dibujante y director de cine. En su niñez y adolescencia, junto con compañeros de colegio, dibujaban cómics y hacían breves películas recibiendo influencia e inspiración de otros artistas, tales como John Lennon, cuyos libros contienen dibujos que el propio Matt pretendió imitar. Su interés por las caricaturas le llevó a desarrollar la habilidad de dibujar durante las horas de clase sin mirar el papel, de modo que pareciera que prestaba atención a los docentes (Robinson, 2009). Con el paso de los años, ya en Los Ángeles y luego de varios trabajos frustrados, Groening creó la serie televisiva Los Simpson, que aún se encuentra emitiendo su trigésima segunda temporada. En este ejemplo podemos vislumbrar la influencia de familiares, amigos y personas famosas en la trayectoria de aprendizaje del dibujante y un progresivo acercamiento a las tecnologías como herramienta para la creación de dibujos y animaciones.

Al igual que Groening, en la actualidad, los jóvenes aprenden de muy diversas maneras, en contextos diferentes y utilizando variadas herramientas, entre las cuales las tecnologías adquieren predominancia. La apertura a nuevos modos de aprendizaje flexible viene dada, en parte, por las nuevas tecnologías móviles y los recursos digitales existentes en la actualidad (Maina y García, 2016).

\section{La apertura a nuevos modos de aprendizaje flexible viene dada, en parte, por las nuevas tecnologías móviles y los recursos digitales existentes en la actualidad (Maina y García, 2016)}

El presente trabajo expone los resultados obtenidos a partir de un cuestionario sobre tecnologías digitales aplicado a estudiantes de educación secundaria. El objetivo del mismo se vinculó a ampliar el conocimiento acerca de las aplicaciones, plataformas web y tecnologías digitales que emplean los estudiantes dentro y fuera de las instituciones escolares.

El interés por ello reside en la importancia de reconocer la gran cantidad de actividades de aprendizaje que se producen en estos contextos, las cuales posibilitan que los estudiantes creen y gestionen sus propias trayectorias (Saadatmand y Kumpulainen, 2012).

En este escrito se presentará, en primer lugar, un desarrollo teórico acerca de las ecologías de aprendizaje y de las TIC y su vinculación con el aprendizaje informal, como conceptos que dan sustento y justifican la importancia del trabajo. En segundo lugar, se expondrán 
aspectos metodológicos dentro de los cuales se detallarán las características del cuestionario sobre tecnologías digitales utilizado. Luego, el apartado de resultados presentará los principales datos obtenidos del instrumento utilizado, organizando el análisis en cuatro categorías. Finalmente, se presentarán algunas discusiones y conclusiones derivadas del escrito.

\section{Ecologías de aprendizaje}

Al considerar los diferentes contextos en los que las personas participan y aprenden, adquiere relevancia el concepto de «ecologías de aprendizaje», el cual es útil para comprender los aprendizajes mediados por tecnologías y la interconexión entre entornos (Espósito et al., 2015).

Barron (2006b) define las «ecologías de aprendizaje» como entidades dinámicas caracterizadas por un conjunto de contextos -formales, no formales y/o informales- que brindan oportunidades para el aprendizaje, cada uno de los cuales contiene ciertas actividades, recursos, relaciones e interacciones inherentes a ellos.

En términos de Espósito et al. (2015), la noción de «ecología» deriva de estudios sobre ecosistemas para referirse a las interrelaciones dinámicas que se establecen entre los organismos y su entorno. Esta metáfora fue adoptada por numerosos autores (Barron y Bell, 2016; Coll, 2013; Jackson, 2013; Maina y García, 2016; Saadatmand y Kumpulainen, 2012, entre otros) a fin de comprender las características que asumen los aprendizajes a lo largo del tiempo y en diferentes entornos, de modo que se consideren las diferencias individuales en las trayectorias de aprendizaje personales (Barron, 2006a; Cortés-González et al., 2016; Hernández-Sellés et al., 2015). En consonancia con el caso que aquí se presenta, una mirada desde las ecologías de aprendizaje destaca el hecho de que los jóvenes participan simultáneamente en muchos contextos e intervienen en diversas prácticas y formas de conocimiento fuera de la escuela (Barron, 2006b). Esta nueva concepción asume que el aprendizaje es multidireccional y multimodal (Saadatmand y Kumpulainen, 2012), por lo que se advierte un distanciamiento de la conceptualización de este proceso vinculado solo a instituciones escolares para comenzar a comprenderlo en una variedad de entornos: el hogar, el lugar de trabajo, la biblioteca, el lugar de juego, el museo, el parque y en las interacciones cotidianas con otros (Barron y Bell, 2016; González-Sanmamed et al., 2018; Martín y Donolo, 2019).

De esta manera, una mirada ecológica habilita a aprender a partir de fuentes, recursos o contextos menos formales (Hernández-Sellés et al., 2015). Así, al hablar de «ecologías de aprendizaje» consideramos tres aspectos: en primer lugar, los límites difusos entre entornos formales, no formales e informales; en segundo lugar, una concepción situada de nuestro aprendizaje; y, en tercer lugar, un alto nivel de personalización de este proceso (Martinenco et al., 2019). 
De acuerdo a lo expresado previamente, la noción de «ecología de aprendizaje» es útil para comprender la hibridación de contextos a partir del uso de TIC, integrando las oportunidades formales, no formales e informales para mejorar el aprendizaje permanente (Sangrà et al., 2011, citado en Espósito et al., 2015) y aumentar las posibilidades de desarrollo y rendimiento (Jackson, 2013).
La noción de «ecología de aprendizaje» es útil para comprender la hibridación de contextos a partir del uso de TIC, integrando las oportunidades formales, no formales e informales para mejorar el aprendizaje permanente

\subsection{TIC y aprendizaje informal}

Con la incorporación de las TIC a la sociedad se modifican múltiples aspectos -incluidos los procesos formativos y educativos-, ya que es preciso adaptarse a las nuevas necesidades de aprendizaje y dar lugar a la formación permanente (Souto-Seijo et al., 2019).

El advenimiento de las TIC cuestiona los formatos de la escuela tradicional, puesto que promueve la ampliación de los ámbitos en los que se localiza el saber, es decir, el conocimiento ya no se encuentra solo en instituciones escolares (Martín, 2002), razón por la que sería interesante que las propuestas didácticas actuales conjugasen el mundo académico y el cotidiano (Cassany y Hernández, 2012).

\section{El uso de las TIC involucra un conjunto de aprendizajes informales en la cotidianidad de los jóvenes, donde la colaboración con otros es central (Buckingham, 2006)}

\section{La facilidad de transportar los dispositivos móviles posibilita que los estudiantes aprendan más allá de los límites temporales y espaciales de la institución educativa}

Como propone Buckingham (2006), el uso de las TIC involucra un conjunto de aprendizajes informales en la cotidianidad de los jóvenes, donde la colaboración con otros es central. Por este motivo, ya no es posible desconocer el entorno personal de aprendizaje del que habla Cassany (2015): ese conjunto de recursos de aprendizaje propio que cada uno utiliza fuera del aula, promovido en gran medida por los dispositivos móviles -tablets, teléfonos móviles, notebooks-, los cuales invitan a aprender a través de múltiples contextos (Bernacki et al., 2020; Mayer, 2020). La facilidad de transportar los dispositivos móviles posibilita que los estudiantes aprendan más allá de los límites temporales y espaciales de la institución educativa. Así lo ilustran los autores

con ejemplos para un mejor entendimiento: se habilita el aprendizaje formal en contextos informales -por ejemplo, participando en una clase virtual desde el autobús-, el aprendizaje informal en contextos informales -por ejemplo, conociendo normas de interacción social a 
través de juegos comunitarios- o el aprendizaje informal en contextos formales -por ejemplo, realizando aprendizaje colaborativo en el aula- (Bernacki et al., 2020).

Los «aprendizajes informales», entendidos como aquellos que ocurren indiferenciada y subordinadamente a otros procesos sociales (Trilla et al., 1993, citado en Martín y Donolo, 2019), derivan del interés voluntario de las personas y su evaluación se centra en valorar la comprensión, la creatividad, la innovación y el progreso de los aprendices (Barron y Bell, 2016; Rogoff et al., 2016). En consonancia con Martín y Donolo (2019), podemos expresar entonces que el aprendizaje informal excede a las instituciones, currículos y programas educativos formales y no formales, atravesando la vida de las personas en todos sus aspectos.

\section{Material y métodos}

En la actualidad se encuentra en desarrollo un estudio vinculado a ecologías de aprendizaje, TIC y emociones que está basado en una metodología de diseño, la cual posee la característica de incorporar experiencias participativas (Gutiérrez y Penuel, 2014) para mejorar las prácticas educacionales (Anderson y Shattuck, 2012; Rinaudo y Donolo, 2010).

Con el objetivo de reconocer el uso de redes, aplicaciones y plataformas web utilizados por un grupo de estudiantes, se diseñó el cuestionario sobre tecnologías digitales.

\subsection{Participantes}

Durante el año 2019, respondieron al mencionado cuestionario 63 estudiantes de quinto y sexto año de educación secundaria que cursaron el espacio curricular Lengua y Literatura en dos instituciones de una localidad de la provincia de Córdoba (Argentina). Del total de participantes, 26 estudiantes cursaban quinto año de Orientación en Ciencias Naturales y 37 cursaban sexto año de Orientación en Ciencias Sociales. La edad promedio de los participantes osciló entre un rango de 16 a 18 años.

\subsection{Instrumento}

El cuestionario sobre tecnologías digitales es un instrumento que se contesta de manera anónima, creado en el marco de un proyecto de investigación mayor, con la intención de ampliar el conocimiento acerca de cuáles son las redes, aplicaciones y plataformas web usadas por los estudiantes y cómo y para qué las emplean.

El conjunto de apartados e ítems que conformó este cuestionario autoadministrado fue seleccionado a partir de las observaciones de clase y de la bibliografía disponible acerca 
de tecnologías y contextos de aprendizaje informales (Alves da Silva y Ferreira, 2016; Cassany, 2015; Domínguez y López, 2015; Pedrero-Esteban et al., 2019). Para determinar su validez, el cuestionario fue revisado por cuatro especialistas en el área de la psicología educativa y la tecnología educativa.

En la versión definitiva, el instrumento contiene cuatro apartados, cada uno de los cuales incluye una serie de ítems a los que los estudiantes debían responder con una cruz, admitiéndose una respuesta por fila. En el primer apartado se pregunta al estudiante si posee teléfono móvil propio y, en caso de responder afirmativamente, para qué lo utiliza; esta es la única sección del instrumento de respuesta abierta. El segundo apartado pretende conocer con qué frecuencia los estudiantes emplean ciertas redes y aplicaciones; para ello se brinda un cuadro de doble entrada en el que consta un listado de 23 ítems -por ejemplo, Pinterest, Facebook, Instagram, WhatsApp, Snapchat, Skype, Canva, Netflix, Google Drive, entre otras- y la frecuencia de uso para que el estudiante marque lo que desee.

Cuadro 1. Ejemplo de ítems del apartado 2 del cuestionario. Modalidad de puntuación (de 1 [no lo utilizo] a 4 [lo utilizo todos los días])

\begin{tabular}{|c|c|c|c|c|}
\hline $\begin{array}{c}\text { Redes y } \\
\text { aplicaciones }\end{array}$ & No lo utilizo & $\begin{array}{l}\text { Lo utilizo poco } \\
\text { (1 o } 2 \text { días } \\
\text { a la semana) }\end{array}$ & $\begin{array}{l}\text { Lo utilizo mucho } \\
\text { (entre } 3 \text { y } 6 \text { días } \\
\text { a la semana) }\end{array}$ & $\begin{array}{l}\text { Lo utilizo } \\
\text { todos los días }\end{array}$ \\
\hline
\end{tabular}

Pinterest

Google Drive

Fuente: elaboración propia.

En el tercer apartado, se presentan 23 actividades con la finalidad de que los participantes respondan con qué frecuencia utilizan las tecnologías en su domicilio para resolverlas. Algunas de las actividades que conforman los ítems son «Realizo las tareas», «Participo en redes sociales para tareas del colegio», «Miro tutoriales para aprender a hacer algo», «Escucho música», entre otras.

Cuadro 2. Ejemplo de un ítem del apartado 3 del cuestionario. Modalidad de puntuación (de 1 [nunca] a 4 [todos los días])

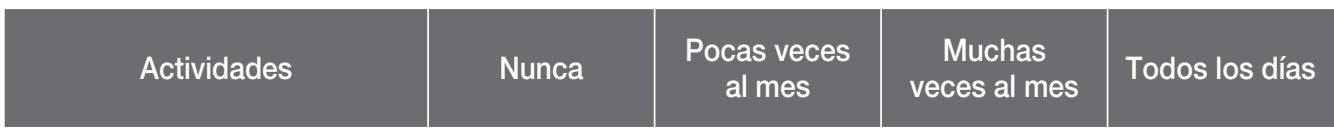

Participo en redes sociales para

tareas del colegio. 
Finalmente, el cuarto apartado solicita a los estudiantes que marquen el nivel de conformidad que poseen respecto a una serie de 15 afirmaciones vinculadas a la realización de actividades mediante tecnologías en las clases. En general, las afirmaciones se refieren a los modos en que los estudiantes llevan a cabo tareas durante las clases -por ejemplo, "Cuando tengo que escribir, prefiero hacerlo en el ordenador»-y sus percepciones respecto a ello -por ejemplo, «Las actividades con tecnologías me resultan más fáciles» o «Las tecnologías me permiten obtener mejores resultados en los trabajos de la escuela»-.

Cuadro 3. Ejemplo de un ítem del apartado 4 del cuestionario. Modalidad de puntuación (de 1 [completamente en desacuerdo] a 5 [totalmente de acuerdo])

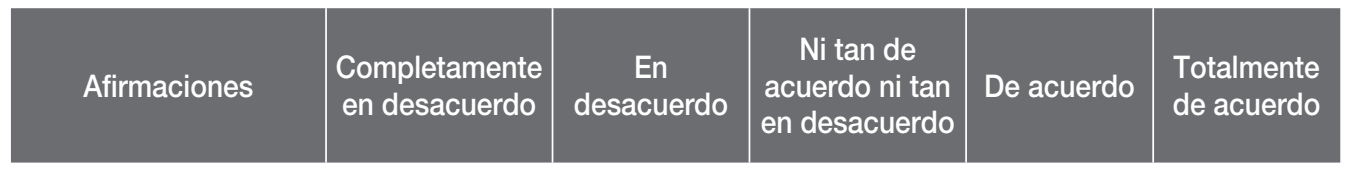

Me gusta traer el telé-

fono móvil a la escuela.

Fuente: elaboración propia.

\subsection{Procedimientos}

El cuestionario sobre tecnologías digitales fue administrado colectivamente y de forma impresa en el mes de octubre de 2019 en dos instituciones de educación secundaria. Al inicio de la clase seleccionada, se les explicó a los participantes las características y objetivos del mismo, su carácter voluntario y la confidencialidad de los datos. El tiempo promedio de respuesta fue de 15 minutos.

Los datos obtenidos del cuestionario se analizaron con procedimientos estadísticos descriptivos, tales como cálculos de media, mediana y desviación estándar. Para ello se utilizó el software Statistical Package for the Social Sciences (SPSS, versión 20).

\section{Análisis y resultados}

Los resultados se presentan en cuatro apartados, cada uno de los cuales corresponde a una categoría derivada de una consigna del cuestionario sobre tecnologías digitales. Las categorías que se considerarán son usos generales del teléfono móvil, empleo de aplicaciones y redes, uso de tecnologías en el hogar y uso de tecnologías en las clases. 


\subsection{Usos generales del teléfono móvil}

La categoría refiere al empleo que los participantes realizan de sus teléfonos móviles y las acciones que desarrollan utilizando los mismos.

De los 63 estudiantes que respondieron al cuestionario, solo el 1,26\% manifestó no poseer teléfono móvil propio. Esta tendencia va en la línea de lo expuesto por el Instituto Nacional de Estadística y Censos (INDEC, 2019) acerca del uso de las TIC en el cuarto trimestre de 2018 en Argentina, ya que el instituto señala que el 88,60\% de las personas entre 13 y 17 años utiliza teléfonos móviles. Los restantes participantes del estudio dieron a conocer para qué emplean sus teléfonos. En este punto cabe aclarar que la mayoría de ellos menciona más de un uso.

En general, las respuestas del cuestionario dejan entrever que los estudiantes usan fundamentalmente sus teléfonos móviles para actividades no escolares. El empleo de las redes sociales también ocupa un lugar central en las respuestas y, si bien advertimos que en los últimos tiem-

Las respuestas del cuestionario dejan entrever que los estudiantes usan fundamentalmente sus teléfonos móviles para actividades no escolares pos encontramos usos educativos de las redes, las expresiones plasmadas en el cuestionario no se vinculan a este modo de empleo de las mismas. En gran medida, los estudiantes destacan que emplean sus teléfonos para mantener contactos con sus familiares y/o amigos y para buscar información. Es interesante advertir respecto a este último punto que, aunque ciertos estudiantes no aclaran el tipo de datos que pretenden encontrar y con qué objetivo, otros explican que lo hacen para estar al corriente de las noticias actuales. Este último caso se percibe exclusivamente en los participantes que cursan sexto año de la rama de Ciencias Sociales. Otro uso no escolar del teléfono móvil que los participantes mencionan asiduamente está relacionado con el entretenimiento a partir de actividades como escuchar música, leer, ver series o películas, jugar con videojuegos, entre otras.

Finalmente, en menor medida, los estudiantes vinculan el uso del teléfono con actividades escolares, sin profundizar demasiado y expresándose de manera global al decir, por ejemplo, «para realizar actividades de la escuela».

En concordancia con lo analizado, una investigación llevada a cabo con estudiantes españoles advirtió acerca del uso del teléfono personal fundamentalmente para mensajería instantánea (81,70\%), el acceso a redes sociales $(77,50 \%)$, el consumo de música $(65,20 \%)$ y el uso de juegos (52,20\%) (Reolid, 2018). En Latinoamérica parece que la tendencia hacia la comunicación se mantiene, ya que un estudio con jóvenes mexicanos de 20 años de edad promedio ha permitido constatar que más del $80 \%$ de los participantes considera muy importante utilizar WhatsApp para comunicarse con su familia y amigos (Gutiérrez-Rentería et al., 2017). También con estudiantes de educación secundaria a distancia en un contexto rural de México se notó la importancia de mantener interacciones y vínculos afectivos a través de 
diversas redes y aplicaciones, como, por ejemplo, Google Classroom, Facebook y WhatsApp (Martínez-Rodríguez y Benítez-Corona, 2020).

En este apartado ha sido posible vislumbrar el modo en que las tecnologías repercuten en la vida cotidiana de los estudiantes en sus diferentes dimensiones (vinculadas a lo académico o no) y en los variados contextos en los que interactúan, como el hogar o la escuela, por ejemplo. No podemos olvidar además que en todos estos entornos y actividades se producen aprendizajes e interacciones, algunos más formales y otros más informales, pero todos ellos son la base para el aprendizaje permanente (Adell y Castañeda, 2010).

\subsection{Empleo de aplicaciones y redes}

Los resultados de este apartado pretenden llevar a cabo una enumeración de las redes sociales, de las aplicaciones y de las páginas web más utilizadas por los participantes y de aquellas otras que emplean menos asiduamente. A continuación, en el cuadro 4, se muestran los principales resultados obtenidos en este sentido. Posteriormente, en la figura 1, se visualiza con mayor claridad cuáles fueron las redes, las aplicaciones y las páginas web más y menos empleadas por los estudiantes.

Cuadro 4. Media y desviación estándar del empleo de aplicaciones y redes

\begin{tabular}{|c|c|c|}
\hline Aplicaciones y redes & Media & Desviación estándar \\
\hline WhatsApp & 3,90 & 0,46 \\
\hline Instagram & 3,84 & 0,44 \\
\hline YouTube & 3,48 & 0,73 \\
\hline Netflix & 2,97 & 1,09 \\
\hline Twitter & 2,54 & 1,36 \\
\hline Google Drive & 2,40 & 1 \\
\hline Telegram & 1,05 & 0,37 \\
\hline Flickr & 1 & 0 \\
\hline Issuu & 0,98 & 0,33 \\
\hline
\end{tabular}


Figura 1. Media obtenida en redes y aplicaciones utilizadas con más y menos asiduidad

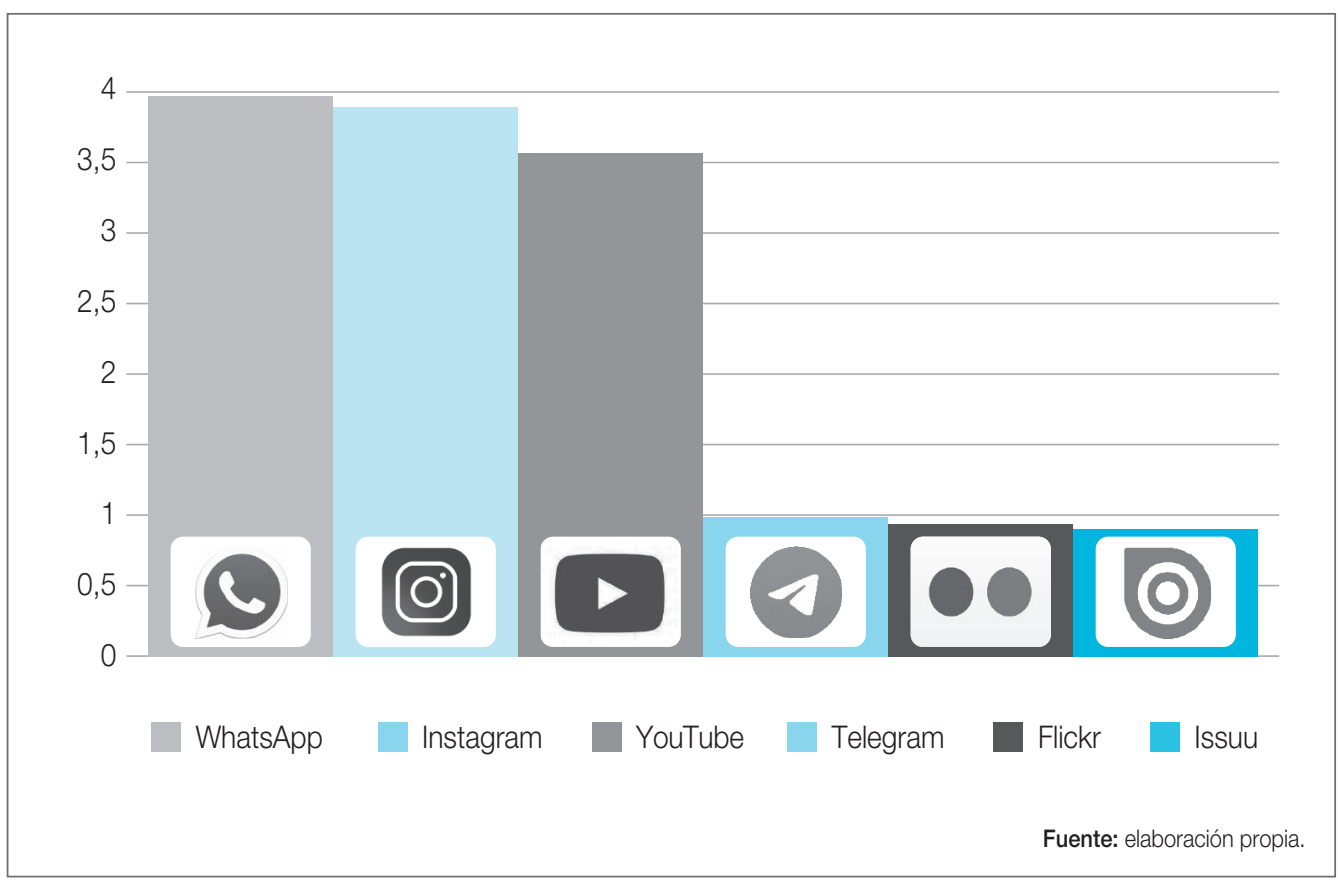

Se puede observar que el grupo participante $(N=63)$ utiliza con amplia frecuencia WhatsApp $(M=3,90)$, Instagram $(M=3,84)$ y YouTube $(M=$ $=3,48)$. Estos valores nos permiten comprender que estas aplicaciones son muy utilizadas -entre 3 y 6 días a la semana- y que algunos estudiantes las emplean todos los días (sobre todo WhatsApp).

En cambio, de acuerdo a las respuestas de los participantes, Telegram $(M=1,05)$, Flickr $(M=1)$ e Issuu $(M=0,98)$ son de uso menos frecuente e incluso en varios casos no se utilizan en ninguna situación.

Se puede observar que el grupo
participante $(N=63)$ utiliza con
amplia frecuencia WhatsApp
$(M=3,90)$, Instagram $(M=3,84)$
y YouTube $(M=3,48)$. Estos
valores nos permiten comprender
que estas aplicaciones son muy
utilizadas -entre 3 y 6 días a la
semana-y que algunos estudiantes
las emplean todos los dias (sobre
todo WhatsApp)

Se puede observar que el grupo participante $(N=63)$ utiliza con amplia frecuencia WhatsApp $(M=3,90)$, Instagram $(M=3,84)$ y You'Tube $(M=3,48)$. Estos valores nos permiten comprender que estas aplicaciones son muy utilizadas -entre 3 y 6 días a la semana- y que algunos estudiantes todo WhatsApp)

Si analizamos las aplicaciones más utilizadas, encontramos un servicio de mensajería, una red social y una página web de vídeos. WhatsApp es una aplicación de mensajería de gran popularidad y con gran adhesión por parte de los participantes del estudio, a diferencia de Telegram -otro servicio de mensajería-, que tiende a no ser utilizado por ellos. Aunque ambas aplicaciones permiten el envío de mensajes de texto y multimedia -vídeos, imágenes y audio- y la realización de videollamadas, la principal diferencia entre ambas reside en la 
posibilidad que ofrece Telegram para enviar mensajes secretos. La segunda aplicación utilizada con mayor asiduidad por parte de los estudiantes es Instagram, una red social cuyo objetivo central es que el usuario comparta fotografías y vídeos con sus seguidores. Instagram posee en común con Flickr -una de las menos utilizadas- el predominio de la imagen, aunque esta última aplicación posee más posibilidades de edición de imágenes. Es importante advertir que, aunque ambas aplicaciones son gratuitas, la versión libre Flickr posee varias limitaciones y menor calidad. La tercera aplicación más empleada por el grupo participante es YouTube, un sitio web que permite compartir y visualizar vídeos de diverso tipo y duración.

Es interesante destacar que tanto WhatsApp como Instagram y YouTube están disponibles para su descarga en el teléfono móvil en español y son de uso gratuito, aunque el sitio web de vídeos también posee su opción de paga. Respecto de Issuu, la aplicación menos empleada por los participantes del estudio, consiste en una página web que permite visualizar materiales digitalizados, tales como libros, revistas o periódicos, sin necesidad de descargar el documento. Por los resultados obtenidos, tendemos a pensar que no sería el sitio web que los estudiantes prefieren para la búsqueda de información mencionada en el apartado anterior. En una posición intermedia, según las respuestas de los estudiantes, encontramos a Netflix $(M=2,97)$, Twitter $(M=2,54)$ y Google Drive $(M=2,40)$ como aquellas aplicaciones y sitio web que emplearían algunos días a la semana. Nuevamente encontramos consistencia con los resultados previos, al tratarse las dos primeras de aplicaciones para entretenimiento y red social, respectivamente; por su parte, Google Drive correspondería al sitio web de uso académico que los estudiantes emplean con más frecuencia (entre 2 y 3 días a la semana).

En general, las aplicaciones más utilizadas coinciden con los resultados hallados por Reolid (2018), quien realizó una investigación con estudiantes de entre 11 y 20 años. En este estudio se pudo determinar que las tecnologías empleadas con mayor frecuencia por los participantes fueron WhatsApp $(77,10 \%)$, redes sociales $(70,10 \%)$ y aplicaciones para escuchar música $(66,60 \%)$. En función de lo expresado hasta el momento podríamos pensar, de acuerdo con Alves da Silva y Ferreira (2016), que una razón fundamental para emplear plataformas en línea y redes sociales es mantener la comunicación con la familia, los amigos y el mundo que los rodea.

\author{
Una razón fundamental para \\ emplear plataformas en línea \\ y redes sociales es mantener la \\ comunicación con la familia, los \\ amigos y el mundo que los rodea
}

\subsection{Uso de tecnologías en el hogar}

En esta sección se presentan los resultados obtenidos en el tercer apartado del cuestionario sobre tecnologías digitales, que se orientó a conocer con qué frecuencia los estudiantes utilizaban las tecnologías en sus hogares para realizar una serie de actividades. En el cuadro 5 se presentan los principales resultados. 
Cuadro 5. Media y desviación estándar del uso de tecnologías en el hogar

\begin{tabular}{|c|c|c|}
\hline Actividades & Media & $\begin{array}{l}\text { Desviación } \\
\text { estándar }\end{array}$ \\
\hline Escucho música. & 3,75 & 0,62 \\
\hline $\begin{array}{l}\text { Me comunico por teléfono móvil con mis compañeros } \\
\text { de colegio. }\end{array}$ & 3,38 & 0,85 \\
\hline $\begin{array}{l}\text { Participo en la producción de tareas o trabajos mediante } \\
\text { documentos compartidos. }\end{array}$ & 2,54 & 0,71 \\
\hline Miro tutoriales para aprender a hacer algo. & 2,33 & 1,03 \\
\hline Busco recetas. & 2,05 & 0,99 \\
\hline Utilizo aplicaciones específicas para cada materia. & 1,56 & 0,79 \\
\hline Participo en blogs o foros. & 1,19 & 0,61 \\
\hline Hago cálculos con Excel. & 1,13 & 0,42 \\
\hline
\end{tabular}

Al analizar las puntuaciones que este grupo de estudiantes $(N=63)$ obtuvo en el apartado 3 del cuestionario, es posible visualizar dos actividades fundamentales para las que los participantes utilizan las tecnologías en los hogares: escuchar música $(M=3,75)$ y comunicarse por teléfono móvil con sus compañeros $(M=3,38)$.

Los dos puntajes arrojados indican que ambas tareas se realizarían con tecnologías de manera frecuente, casi todos los días; es decir, la amplia mayoría de los participantes emplea las tecnologías en sus hogares para desarrollar actividades vinculadas con lo analizado en los apartados anteriores.

Esto implica desempeñar tareas referidas a dos de las aplicaciones empleadas con mayor asiduidad -WhatsApp y YouTube- y a los principales usos de los teléfonos móviles mencionados por los estudiantes en el primer apartado del cuestionario -comunicación y entretenimiento-.

Por el contrario, los participantes del estudio expresan que en muy pocas ocasiones -y nunca en algunos casos- usan la tecnología en sus domicilios para realizar actividades como calcular con Excel $(M=1,13)$, participar en blogs o foros $(M=1,19)$ y usar aplicaciones específicas para cada materia $(M=1,56)$. 
El presente apartado nos permite comprender que, en general, los usos de las tecnologías que los estudiantes realizan en sus hogares no tendrían vinculación directa con actividades académicas, sino, fundamentalmente, con tareas de comunicación y recreación. En este sentido, un estudio realizado con estudiantes de segundo año de educación secundaria acerca de las potencialidades de Insta-

Los usos de las tecnologías que los estudiantes realizan en sus hogares no tendrían vinculación directa con actividades académicas, sino, fundamentalmente, con tareas de comunicación y recreación gram para la enseñanza en Biología mostró la sorpresa inicial de los participantes al utilizar una red social con fines educativos y la expectativa por su empleo como recurso didáctico (Zuber et al., 2020). Como podemos percibir, aún existen distancias y discontinuidades entre los aprendizajes dentro y fuera de la escuela, incluyendo los recursos que se utilizan, las herramientas cognitivas que se ponen en práctica y el tipo de desempeño que se prioriza -individual o colectivo-, entre otros aspectos (Resnick, 1987).

\subsection{Uso de tecnologías en las clases}

En el presente apartado se presentan los resultados vinculados al nivel de conformidad que los estudiantes presentan respecto a una serie de afirmaciones sobre el uso de tecnologías en las clases. Esta categoría deriva de los datos obtenidos en el cuarto apartado del cuestionario sobre tecnologías digitales, cuyos ítems responden a cinco dimensiones del uso de tecnologías en las clases: académica, cognitiva, afectiva, social y técnica. A continuación, se presenta el cuadro 6 , que contiene los principales resultados.

Cuadro 6. Media y desviación estándar del uso de tecnologías en las clases

\begin{tabular}{|c|c|c|}
\hline Actividades & Media & Desviación estándar \\
\hline Me gusta traer el teléfono móvil a la escuela. & 4,78 & 0,45 \\
\hline $\begin{array}{l}\text { Cuando necesito buscar una información, recurro en } \\
\text { primer lugar a las tecnologías. }\end{array}$ & 4,62 & 0,55 \\
\hline $\begin{array}{l}\text { Las tecnologías me permiten realizar mis trabajos de } \\
\text { modo más creativo. }\end{array}$ & 4,19 & 1,04 \\
\hline $\begin{array}{l}\text { Cuando tengo que escribir, prefiero hacerlo en el orde- } \\
\text { nador. }\end{array}$ & 4,16 & 1,03 \\
\hline Las actividades con tecnologías me resultan más fáciles. & 4,13 & 1,02 \\
\hline
\end{tabular}




\begin{tabular}{|c|c|c|}
\hline Actividades & Media & Desviación estándar \\
\hline \multicolumn{3}{|l|}{4} \\
\hline $\begin{array}{l}\text { Las tecnologías me permiten obtener mejores resulta- } \\
\text { dos en los trabajos de la escuela. }\end{array}$ & 4,08 & 0,78 \\
\hline $\begin{array}{l}\text { Las actividades con tecnologías me resultan más diver- } \\
\text { tidas. }\end{array}$ & 3,81 & 0,91 \\
\hline $\begin{array}{l}\text { Considero que trabajo mejor en grupo si utilizamos tec- } \\
\text { nologías. }\end{array}$ & 3,75 & 1,10 \\
\hline $\begin{array}{l}\text { Me interesan más las actividades que incluyen tecno- } \\
\text { logías. }\end{array}$ & 3,71 & 1,05 \\
\hline $\begin{array}{l}\text { Encuentro el estudio más sencillo cuando utilizo tecno- } \\
\text { logías. }\end{array}$ & 3,60 & 1,35 \\
\hline $\begin{array}{l}\text { Me gusta leer desde el teléfono móvil, el ordenador o } \\
\text { la tablet. }\end{array}$ & 3,37 & 1,31 \\
\hline $\begin{array}{l}\text { En la mayoría de las clases de Lengua utilizamos tecno- } \\
\text { logías. }\end{array}$ & 3,27 & 0,97 \\
\hline $\begin{array}{l}\text { En las clases aprendo a usar nuevas tecnologías o apli- } \\
\text { caciones. }\end{array}$ & 3,03 & 1,06 \\
\hline $\begin{array}{l}\text { Cuando usamos tecnologías, la profesora explica su } \\
\text { funcionamiento. }\end{array}$ & 2,97 & 1,17 \\
\hline Me pone nervioso trabajar con tecnologías. & 1,49 & 0,80 \\
\hline
\end{tabular}

En consonancia con lo presentado en el cuadro 6, los resultados muestran que los participantes $(N=63)$ están de acuerdo con tres afirmaciones respecto al uso de las tecnologías en las clases: les gusta llevar el teléfono móvil a la escuela $(M=4,78)$, cuando necesitan buscar información recurren en primer lugar a las tecnologías $(M=4,62)$ y, en el momento de escribir, prefieren hacerlo en el ordenador $(M=4,16)$. Estas dos últimas afirmaciones, vinculadas a una dimensión cognitiva, nos muestran las prioridades de los estudiantes frente a la posibilidad de elegir diferentes modos de llevar a cabo una tarea escolar, ya sea buscar información o escribir. También encontramos aquí una correlación con un estudio realizado con estudiantes universitarios de Puerto Rico, quienes utilizan internet como su primera opción para la búsqueda de información y no la biblioteca física (Soto, 2019). 
Por el contrario, el nivel de conformidad de los participantes con la afirmación vinculada a sentir nervios en el momento de trabajar con tecnologías es bajo $(M=1,49)$, lo que nos permitiría pensar que estos estudiantes no experimentan este tipo de estados emocionales negativos al trabajar con TIC. Otros ítems del cuestionario que se refieren también a la dimensión afectiva a la hora de realizar actividades con tecnologías indicarían una tendencia similar, al mostrar que los participantes perciben como divertidas las actividades que emplean TIC $(M=$ $=3,81$ ) y que poseen gran interés por las tareas que involucran este tipo de recursos $(M=3,71)$. Y, como se explicitó en el párrafo anterior, les gusta llevar el teléfono móvil a la escuela $(M=4,78)$.

Adquiriendo valores intermedios encontramos afirmaciones relativas a aprender a usar nuevas tecnologías en clase $(M=3,03)$ y a que, al utilizar tecnologías, la profesora explica su funcionamiento $(M=2,97)$. Tal vez podríamos pensar, a partir de estos ítems de dimensiones cognitiva y técnica, respectivamente, que en las clases de Lengua y Literatura que conformaron el estudio no se habrían empleado TIC completamente nuevas para los estudiantes o que hubieran requerido una demostración explícita de su uso.

Con puntuaciones un poco mayores, pero sin alcanzar un completo acuerdo por parte de los estudiantes, se encuentran dos afirmaciones vinculadas a las dimensiones académica y social que reconocen que el uso de tecnologías mejoraría los resultados de los trabajos escolares $(M=4,08)$ y el trabajo grupal $(M=3,75)$.

Los límites entre los aprendizajes

formales y no formales se van diluyendo, puesto que los participantes llegan a clase con un bagaje de aprendizajes previos vinculados a la manipulación de tecnologías, al uso de estrategias de búsqueda de información digital [...] y los ponen en juego en las tareas escolares
Este apartado del estudio nos permite comprender cómo los límites entre los aprendizajes formales y no formales se van diluyendo, puesto que los participantes llegan a clase con un bagaje de aprendizajes previos vinculados a la manipulación de tecnologías, al uso de estrategias de búsqueda de información digital, al empleo de softwares de procesamiento de textos, entre otros, y los ponen en juego en las tareas escolares.

\section{Discusión y conclusiones}

De modo general, podemos expresar que el cuestionario sobre tecnologías digitales brindó resultados que permitieron profundizar nuestro conocimiento acerca de aplicaciones, plataformas web, redes sociales y tecnologías que este grupo de estudiantes emplea en su vida cotidiana, tanto dentro como fuera de la institución escolar. Los resultados hallados 
en ese sentido posibilitan vislumbrar la amplia presencia que actualmente poseen las TIC en las actividades diarias de los participantes en el estudio, donde el aprendizaje es un aspecto central.

Así, encontramos un uso generalizado del teléfono móvil en la mayoría de los estudiantes fundamentalmente para comunicarse, entretenerse y participar en redes sociales. Estos modos de emplear el dispositivo móvil coinciden con aquellas aplicaciones que se emplean de manera prioritaria y con las actividades que desempeñan en los hogares apoyados en las tecnologías: WhatsApp, para comunicarse con compañeros de colegio; Instagram, como una las redes sociales de preferencia; y YouTube, como una opción para escuchar música. Específicamente, en el ámbito escolar cobra relevancia la tecnología como primera opción a la que recurren los estudiantes para la búsqueda de información y para la escritura. Por supuesto que encontramos, además, usos específicos en cada estudiante de acuerdo a sus intereses, de modo que van conformando sus propias trayectorias de aprendizaje individuales. Aquí es interesante advertir que las aplicaciones más empleadas se vinculan a los usos que los estudiantes hacen de las tecnologías en sus hogares, por lo que podríamos pensar que el dispositivo móvil no es empleado con gran frecuencia en las clases analizadas.

En términos de Adell y Castañeda (2010), estos entornos personales de aprendizaje se van conformando alrededor de tres tipos de herramientas. Una de ellas es la de relación con otros y, en este estudio, encontramos fundamentalmente WhatsApp e Instagram como aplicaciones de mensajería y red social, respectivamente. Otra herramienta alrededor de la cual se configuran los entornos personales de aprendizaje es la de creación y edición de información, como la empleada por algunos estudiantes que utilizan las TIC en sus hogares para realizar tareas mediante documentos compartidos. Finalmente, la herramienta de acceso a la información se detecta en este grupo de estudiantes con la búsqueda de información en sitios de publicaciones o vídeos, como es el caso de YouTube. Como podemos comprender, estas herramientas son socialmente compartidas y se vinculan a situaciones concretas de la vida diaria (Resnick, 1987).

Retomando los supuestos del estudio, nuestros resultados van en la línea de lo detectado por Tabuenca et al. (2013), puesto que la combinación de dispositivos móviles que posibiliten utilizar la variedad de aplicaciones, redes y tecnologías ayudaría a que los estudiantes promuevan su aprendizaje continuo y desarrollen sus propias ecologías de aprendizaje. De esta manera, la tecnología se convierte en un aspecto central de las «ecologías» al conducir a esa difuminación de límites entre los aprendizajes y contextos formales e informales (Maina y García, 2016).
La tecnología se convierte en un aspecto central de las «ecologías» al conducir a esa difuminación de límites entre los aprendizajes y contextos formales e informales (Maina y García, 2016) 
Consideramos que el aporte de este trabajo se orienta a entender que las tecnologías están presentes en la vida cotidiana de los participantes -y también en la nuestra- en sus múltiples aspectos: comunicación, entretenimiento, búsqueda de información, realización de tareas académicas, reconociendo que en todas estas actividades se van construyendo aprendizajes. Por este motivo, a lo largo del estudio destacamos los límites difusos que encontramos entre ocio y trabajo, aprendizajes en el interior y en el exterior de la escuela e, incluso, entre los participantes. En consonancia con lo planteado por Díaz-Barriga (2020), los estudiantes usan mayormente las tecnologías para comunicarse y para utilizar las redes sociales, pero no necesariamente como un recurso de aprendizaje.

Creemos necesario continuar con la investigación y analizar la interacción entre los entornos informales y las instituciones escolares. En este sentido, Shafirova y Cassany (2017) advierten acerca de la necesidad de vincular los intereses extraescolares de los estudiantes con la educación formal y, en este punto, es donde consideramos interesante alentar la realización de estudios de diseño que contemplen estos aspectos, creando una propuesta didáctica conjuntamente con los docentes para llevar a cabo en las clases (Rinaudo y Donolo, 2010).

\section{Referencias bibliográficas}

Adell Segura, J. y Castañeda Quintero, L. (2010). Los entornos personales de aprendizaje (PLEs): una nueva manera de entender el aprendizaje. En R. Roig Vila y M. Fiorucci (Eds.), Claves para la investigación en innovación y calidad educativas: la integración de las tecnologías de la información y la comunicación y la interculturalidad en las aulas (pp. 19-30). Marfil; Roma TRE Universita degli Studi.

Alves da Silva, C. y Ferreira, C. (2016). Las redes sociales y el aprendizaje informal de estudiantes de educación superior. Acción Pedagógica, 25, 6-20. https://bit.ly/2ylfAS2

Anderson, T. y Shattuck, J. (2012). Designbased research: a decade of progress in education research? Educational Researcher, 41(1), 16-25. https://doi.org/10.3102/00131 $89 \times 11428813$
Barron, B. (2006a). Configurations of learning settings and networks: implications of a learning ecology perspective. Human Development, 49, 229-231. https://doi.org/10.1159/ 000094370

Barron, B. (2006b). Interest and self-sustained learning as catalysts of development: a learning ecology perspective. Human Development, 49, 193-224. https://doi.org/10.1159/ 000094368

Barron, B. y Bell, P. (2016). Learning environments in and out of school. En L. Corno y E. Anderman (Eds.), Handbook of Educational Psychology (pp. 323-335). Routledge.

Bernacki, M., Greene, J. y Crompton, H. (2020). Mobile technology, learning, and achievement: advances in understanding and measuring the role of mobile technology in education 
Contemporary Educational Psychology, 60, 1-8. https://doi.org/10.1016/j.cedpsych.2019 101827

Buckingham, D. (2006). Educación en medios: alfabetización, aprendizaje y cultura contemporánea. Paidós Comunicación.

Cassany, D. (2015). Redes sociales para leer y escribir. En G. Bañales, N. Vega López y M. Castelló (Eds.), Enseñar a leer y escribir en la educación superior: propuestas educativas basadas en la investigación (pp. 187-208). Fundación SM México.

Cassany, D. y Hernández, D. (2012). ¿Internet: 1; Escuela: 0? CPU-e. Revista de Investigación Educativa, 14, 126-141. https://doi.org/10.25 009/cpue.v0i14.32

Cobo Romani, C. y Moravec, J. (2011). Aprendizaje invisible: hacia una nueva ecología de la educación. Collecció Transmedia XXI. Publicacions i Edicions de la Universitat de Barcelona.

Coll, C. (2013). El currículo escolar en el marco de la nueva ecología del aprendizaje. Aula de Innovación Educativa, 219, 31-36. https://bit. ly/2ZoaZtx

Cortés-González, P., Leite-Méndez, A., RivasFlores, J. I., García-López, M. y Cortés-González, A. (2016). Estudio etnográfico narrativo sobre ecologías del aprendizaje en la Universidad Paulo Freire de la Serranía de Ronda. Investigación Cualitativa en Educación, 1, 623-628. https://doi.org/10.13140/ RG.2.1.4468.8881

Díaz-Barriga, A. (2020). La escuela ausente, la necesidad de replantear su significado. En H. Casanova Cardiel (Coord.), Educación y pandemia: una visión académica (pp. 19-29). Universidad Nacional Autónoma de México, Instituto de Investigaciones sobre la Universidad y la Educación.

Domínguez, F. y López, R. (2015). Uso de las redes sociales digitales entre los jóvenes universitarios en México. Hacia la construcción de un estado del conocimiento
(2004-2014). Revista de Comunicación, 14, 48-69. https://bit.ly/2Xayi7a

Espósito, A., Sangrá, A. y Maina, M. (2015). Emerging learning ecologies as a new challenge and essence for e-learning. The case of doctoral e-researchers. En M. Ally y B. Khan (Eds.), International Handbook of E-learning. Vol. 1 (pp. 331-342). Routledge.

González-Sanmamed, M., Sangra, A., SoutoSeijo, A. y Estévez Blanco, I. (2018). Ecologías de aprendizaje en la era digital: desafíos para la educación superior. Publicaciones, 48(1), 25-45. https://doi.org/10.30827/publicacio nes.v48i1.7329

Gutiérrez, K. y Penuel, W. (2014). Relevance to practice as criterion for rigor. Educational Researcher, 43(1), 19-23. https://doi.org/10. 3102/0013189x13520289

Gutiérrez-Rentería, M. ${ }^{a}$ E., Santana-Villegas, J. C. y Pérez-Ayala, M. (2017). Smartphone: usos y gratificaciones de los jóvenes en México en 2015. Palabra Clave-Revista de Comunicación, 20(1), 47-68. https://doi.org/10.5294/ pacla.2017.20.1.3

Hernández-Selles, N., González-Sanmamed, M. y Muñoz-Carril, P. (2015). El rol docente en las ecologías de aprendizaje: análisis de una experiencia de aprendizaje colaborativo en entornos virtuales. Profesorado. Revista de Currículum y Formación de Profesorado, 19(2), 147-163. https://bit.ly/2TrT8Ov

INDEC. (2019). Acceso y uso de tecnologías de la información y la comunicación. EPH. Cuarto trimestre de 2018. Informes Técnicos, 3(86). https://bit.ly/2AJNPDr

Jackson, N. (2013). The concept of learning ecologies. En N. Jackson y B. Cooper (Eds.), Lifewide Learning, Education \& Personal Development [libro electrónico] (pp. 1-21).

Maina, M. y García, I. (2016). Articulating personal pedagogies through learning ecologies. En B. Gros, Kinshuk y M. Maina (Eds.), The Future of Ubiquitous Learning: Learning Designs for Emerging Pedagogies (pp. 73-94). Lecture Notes in Educational Techno. 
Martín Barbero, J. (2002). La educación desde la comunicación. Grupo Editorial Norma.

Martín, R. B. y Donolo, D. S. (2019). Aprendizajes informales. Perspectivas teóricas y relatos de aprendizajes. IKASTORRATZA. e-Revista de Didáctica, 23, 115-131. https://doi.org/ 10.37261/23_alea/5

Martinenco, R.; Martín, R. B. y García, L. (2019). Ecologías de aprendizaje. Una reflexión sobre el aprendizaje en ambientes diversos. En D. Michelini, S. Otero y M. Crabay (Eds.), Convivir en un mundo con fronteras (pp. 137-139). Del Icalá.

Martínez-Rodríguez, R. y Benítez-Corona, L. (2020). La ecología del aprendizaje resiliente en ambientes ubicuos ante situaciones adversas. Comunicar, 28(62), 43-52. https:// doi.org/10.3916/C62-2020-04

Mayer, R. (2020). Where is the learning in mobile technologies for learning? Contemporary Educational Psychology, 60, 1-3. https://doi. org/10.1016/j.cedpsych.2019.101824

Pedrero-Esteban, L., Barrios-Rubio, A. y MedinaÁvila, V. (2019). Adolescentes, smartphones y consumo de audio digital en la era de Spotify. Comunicar, 27(60), 103-112. https://doi. org/10.3916/C60-2019-10

Reolid Martínez, R. E. (2018). Usos de internet $y$ redes sociales por parte de adolescentes y estudiantes de ciencias de la salud. (Tesis doctoral, Universidad de Castilla-La Mancha). Repositorio https://bit.ly/3e7yz8l

Resnick, L. (1987). Education and Learning to Think. National Academy Press.

Rinaudo, M. ${ }^{a}$ C. y Donolo, D. S. (2010). Estudios de diseño. Una alternativa prometedora en la investigación educativa. RED. Revista de Educación a Distancia, 22, 2-29. https://bit. ly/2TqWqlf

Robinson, K. (2009). El elemento: descubrir tu pasión lo cambia todo. Conecta.

Rogoff, B., Callanan, M., Gutiérrez, K. y Erickson, F. (2016). The organization of informal learning. Review of Research in Education
(RRE), 40, 356-401. https://doi.org/10.3 102/0091732×16680994

Saadatmand, M. y Kumpulainen, K. (2012). Emerging technologies and new learning ecologies: learners' perceptions of learning in open and networked environments. En V. Hodgson, C. Jones, M. de Laat, D. McConnell, T. Ryberg y P. Sloep (Eds.), Proceedings of the 8th International Conference on Networked Learning 2012 (pp. 266-275). Intensive Care Medicine.

Shafirova, L. y Cassany, D. (2017). Aprendiendo idiomas en línea en el tiempo libre. Revista de Estudios Socioeducativos, 5, 49-62. https:// doi.org/10.25267/rev_estud_socioeducati vos.2017.i5.06

Soto Paz, P. A. (2019). Análisis de preferencias en la búsqueda de información; utilización de internet y/o biblioteca física. Caso de estudio en la Universidad de Puerto Rico. MLS-Educational Research, 3(2), 47-64. https://doi. org/10.29314/mlser.v3i2.215

Souto-Seijo, A., Estévez, I., González-Sanmamed, M. y Romero, P. (2019). Technological resources for lifelong learning of teachers in the digital era: an analysis from the learning ecologies. En A. Volungeviciene y A. Szücs (Eds.), EDEN 2019 ANNUAL Conference "Connecting through Educational Technology to Produce Effective Learning Environments». European Distance and E-Learning Network.

Tabuenca, B., Ternier, S. y Specht, M. (2013). Patrones cotidianos en estudiantes de formación continua para la creación de ecologías de aprendizaje. RED. Revista de Educación a Distancia, 37, 1-13. Número especial dedicado a «Aprendizaje ubicuo». https://bit.ly/2W QaEht

Zuber Schachner, S., Vigliecca, F. y Martin, R. (2020). Cierren carpetas y saquen los celulares. Potencialidades de Instagram para la enseñanza de diversidad biológica en segundo año de educación secundaria. Revista de Educación en Biología, 23(1), 21-34. 


\section{ilnicio en febrero y octubre de cada año!}

\section{Magisterio de Educación Infantil}

Hoy en día los centros educativos tienen una imperiosa necesidad de disponer de personal especializado, capaz de hacer frente a las necesidades educativas de la etapa infantil, de acuerdo con los conocimientos ya logrados por las diversas ciencias que hoy se ocupan de los niños en los primeros años de su vida, así como de los logros relativos al desarrollo de la inteligencia, la emocionalidad y la formación de la personalidad temprana, resultantes de estudios recientes sobre el desenvolvimiento de la mente infantil.

\section{Magisterio de Educación Primaria}

Son objetivos de la educación primaria, entre otros: conocer y apreciar los valores y las normas de convivencia, aprender a obrar de acuerdo con ellas, prepararse para el ejercicio activo de la ciudadanía y respetar los derechos humanos, así como el pluralismo propio de una sociedad democrática. También, desarrollar hábitos de trabajo individual y de equipo, de esfuerzo y responsabilidad en el estudio, así como actividades de confianza en uno mismo, sentido crítico, iniciativa personal, curiosidad, interés y creatividad en el aprendizaje.

\section{Menciones en los grados de Magisterio de Educación Infantil y de Magisterio de Educación Primaria}

Mención en Lengua Inglesa

Mención en Pedagogía Terapéutica

Mención en Audición y Lenguaje

Mención en Tecnología Educativa

Mención en Enseñanza de la Religión Católica

\section{Curso de adaptación al grado}

Este curso de adaptación al grado ofrece a los maestros diplomados en la Especialidad de Educación Infantil o Primaria la posibilidad de obtener formación en campos determinados dentro del ejercicio profesional docente en estas etapas, a través de las menciones cualificadoras mencionadas anteriormente.

El objetivo principal del plan de estudios de este curso de adaptación al grado es contribuir a la actualización de la formación de los maestros diplomados. La aplicación de las TIC a la educación y de líneas pedagógicas innovadoras, fruto de la investigación en educación, hacen necesaria la actualización de los conocimientos didácticos de los diplomados y la formación de los maestros en investigación e innovación.

Al finalizar el curso de adaptación se obtiene el título de grado en Magisterio de Educación Infantil o en Magisterio de Educación Primaria.

La docencia en la etapa de educación infanti o primaria es una profesión regulada. Los graduados en Magisterio de Educación Infantil o en Magisterio de Educación Primaria tienen como principal salida profesional el trabajo como profesores en estas etapas, tanto en centros públicos como concertados y privados.

Si bien otras salidas profesionales para estos títulos pueden ser:

- Participación en proyectos educativos de organismos e instituciones (centros culturales, museos, asociaciones, ONG, etc.).

- Centros de educación para adultos.

- Centros de ocio y tiempo libre.

- Participación en programas de extensión educativa (actividades extraescolares, actividades de apoyo, etc.).

- Diseño y elaboración de materiales didácticos.

- Participación en proyectos de atención a la infancia y familiar. 